\title{
Molybdenum(VI) Sequestration Mechanisms During Iron(II)-Induced Ferrihydrite Transformation
}

\author{
Supplemental Information
}

Valerie A. Schoepfer, ${ }^{\dagger},{ }^{*}$ Jullieta E. Lum, ${ }^{\dagger}$ Matthew B. J. Lindsay ${ }^{\dagger}, *$

†Department of Geological Sciences, University of Saskatchewan, Saskatoon, SK, S7N 5E2,

Canada

* Corresponding authors. Tel: +1 3069665693.

E-mail address: valerie.schoepfer@usask.ca (V.A. Schoepfer), matt.lindsay@usask.ca (M.B.J.

Lindsay) 

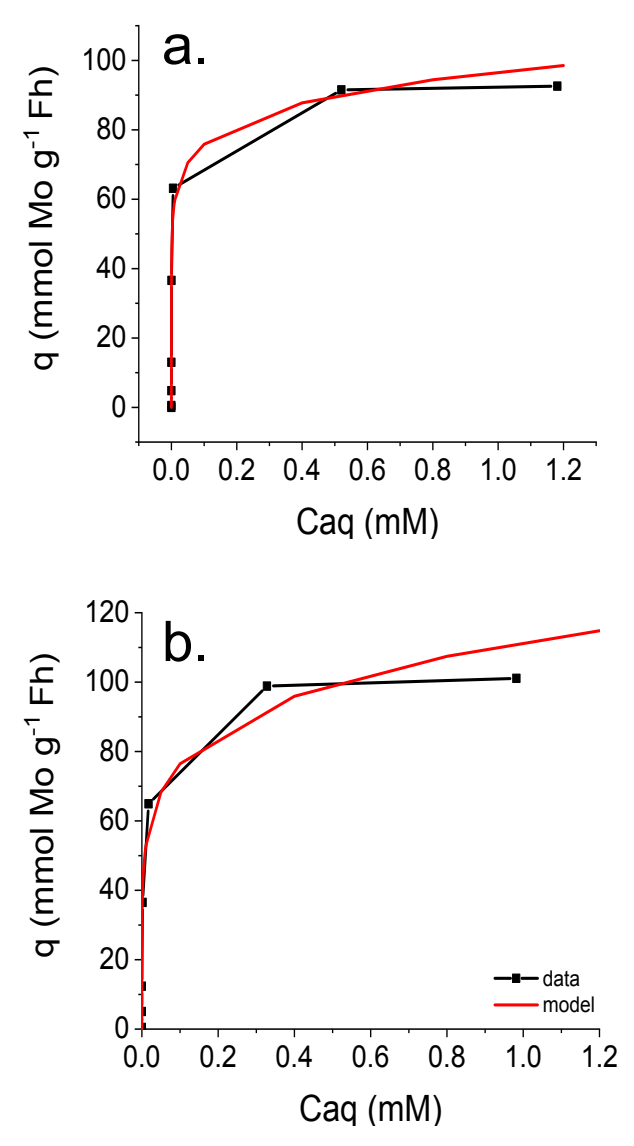

Figure $\mathrm{S} 1$. Freundlich isotherm models for the experiments at a) $\mathrm{pH}_{0} 5.0$ and b) $\mathrm{pH}_{0}$ 6.5. Data is represented in black squares and the fitted model is the red solid line. 

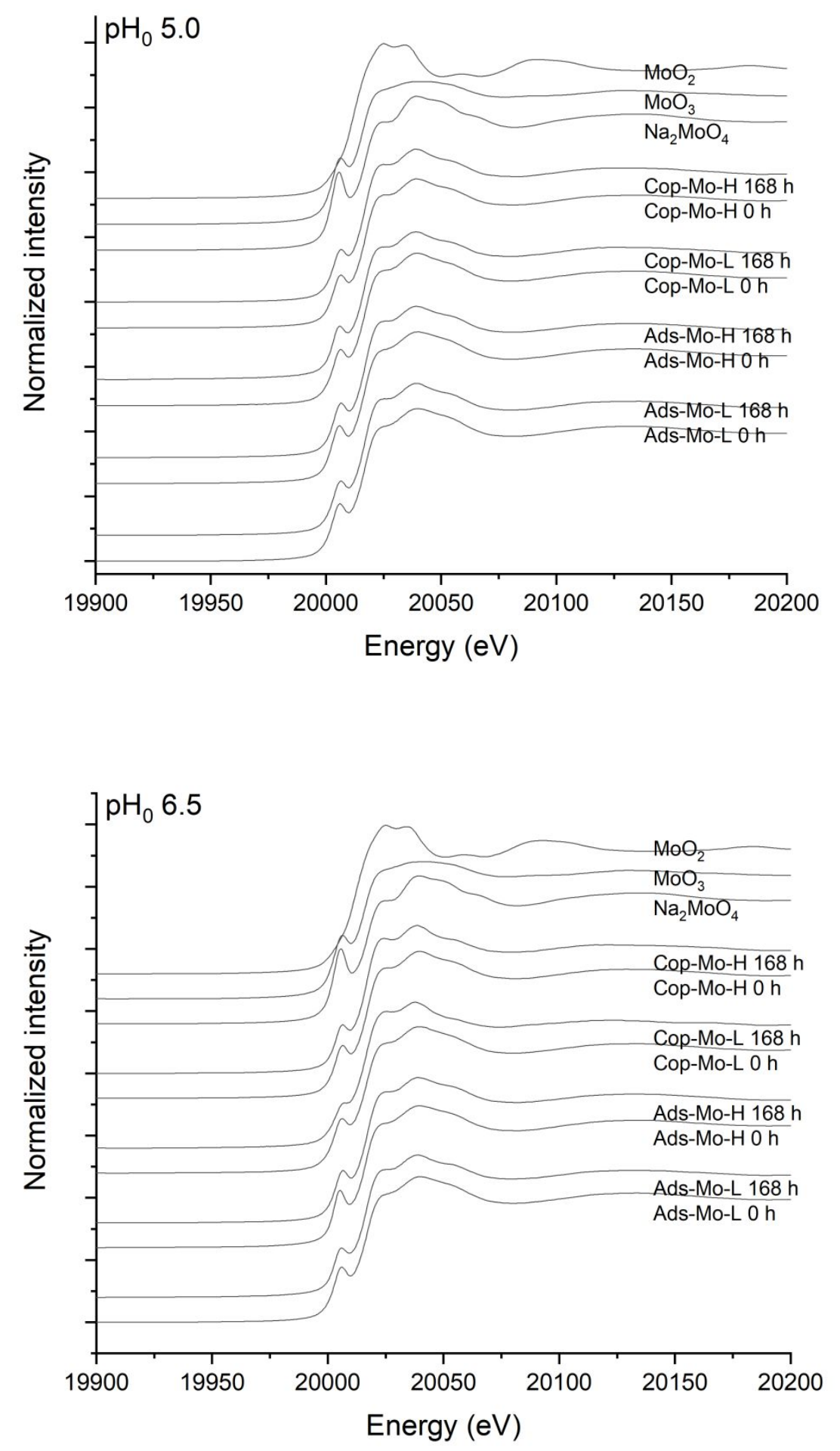

Figure S2. Normalized XANES intensity of the $\mathrm{pH}_{0} 5.0$ experiments (top) and $\mathrm{pH}_{0} 6.5$ experiments (bottom) over the Mo K-edge before and after Fe(II) addition from $19900 \mathrm{eV}$ to $20200 \mathrm{eV}$. Standards include $\mathrm{Na}_{2} \mathrm{MoO}_{4}, \mathrm{MoO}_{3}$, and $\mathrm{MoO}_{2}$. 
Table S1. Modelled EXAFS fitting parameters for $\mathrm{pH}_{0} 5.0$ experiments. Parameters include the amplitude reduction factor which was fixed at 0.82 , the Debye-Waller factor $\left(\sigma^{2}\right)$, interatomic distance $(\mathrm{R})$, coordination number $(\mathrm{CN})$, and energy shift $\left(\Delta \mathrm{E}_{0}\right)$. The fitting range used was $\mathrm{k}=$ 3.0 to $11.0 \AA^{-1}$ and $\mathrm{R}=1.0$ to $4.0 \AA$. Time ' 0 ' indicates the experiments were collected before $\mathrm{Fe}(\mathrm{II})$ addition.

\begin{tabular}{|c|c|c|c|c|c|c|c|c|}
\hline pH & ID & Time & Path & $\mathrm{CN}$ & $\mathbf{R}(\mathbf{A})$ & $\sigma^{2}(\AA)$ & $\Delta \mathbf{E}_{0}$ & R-factor \\
\hline \multirow[t]{2}{*}{5} & Ads-Mo-L & 0 & Mo-O & $3.9(2)$ & $1.739(4)$ & $0.0018(6)$ & 5.32 & 0.004 \\
\hline & & & $\mathrm{Mo}-\mathrm{Fe}_{1}$ & $0.6(6)$ & $2.75(2)$ & $0.009(8)$ & & \\
\hline \multirow[t]{2}{*}{5} & Ads-Mo-L & 168 & $\mathrm{Mo}-\mathrm{O}_{1}$ & $3.2(2)$ & $1.727(5)$ & $0.0020(7)$ & 2.18 & 0.010 \\
\hline & & & $\mathrm{Mo}-\mathrm{Fe}_{2}$ & $0.5(4)$ & $3.15(2)$ & $0.001(4)$ & & \\
\hline \multirow[t]{2}{*}{5} & Ads-Mo-H & 0 & Mo-O & $3.9(2)$ & $1.738(3)$ & $0.0020(6)$ & 5.39 & 0.004 \\
\hline & & & $\mathrm{Mo}-\mathrm{Fe}_{1}$ & $0.6(5)$ & $2.75(2)$ & $0.008(7)$ & & \\
\hline \multirow[t]{2}{*}{5} & Ads-Mo-H & 168 & Mo-O & $3.3(3)$ & $1.728(5)$ & $0.0019(8)$ & 2.78 & 0.008 \\
\hline & & & $\mathrm{Mo}-\mathrm{Fe}_{2}$ & $0.6(5)$ & $3.14(2)$ & $0.002(6)$ & & \\
\hline \multirow[t]{2}{*}{5} & Cop-Mo-L & 0 & $\mathrm{Mo} \mathrm{O}_{1}$ & $3.9(3)$ & $1.739(5)$ & $0.0027(7)$ & 5.18 & 0.008 \\
\hline & & & $\mathrm{Mo}-\mathrm{Fe}_{2}$ & $0.5(4)$ & $3.18(2)$ & $0.001(6)$ & & \\
\hline \multirow[t]{4}{*}{5} & Cop-Mo-L & 168 & Mo-O & $3.6(2)$ & $1.720(4)$ & $0.0026(6)$ & -0.87 & 0.004 \\
\hline & & & $\mathrm{Mo}-\mathrm{O}_{2}$ & $2.6(1.7)$ & $2.23(3)$ & $0.02(1)$ & & \\
\hline & & & $\mathrm{Mo}-\mathrm{Fe}_{2}$ & $0.5(7)$ & $3.13(4)$ & $0.01(1)$ & & \\
\hline & & & Mo-Mo & $0.6(1)$ & $3.34(1)$ & $0.0001(10)$ & & \\
\hline \multirow[t]{2}{*}{5} & Cop-Мo-H & 0 & Mo-O & $3.6(2)$ & $1.730(5)$ & $0.0021(7)$ & 3.21 & 0.006 \\
\hline & & & $\mathrm{Mo}-\mathrm{Fe}_{2}$ & $0.6(4)$ & $3.15(2)$ & $0.001(5)$ & & \\
\hline \multirow[t]{4}{*}{5} & Cop-Мo-H & 168 & $\mathrm{Mo}^{-\mathrm{O}_{1}}$ & $3.4(2)$ & $1.715(4)$ & $0.0018(5)$ & -1.69 & 0.003 \\
\hline & & & Mo- $\mathrm{O}_{2}$ & $1.3(5)$ & $2.23(2)$ & $0.010(6)$ & & \\
\hline & & & $\mathrm{Mo}-\mathrm{Fe}_{2}$ & $0.6(4)$ & $3.10(1)$ & $0.003(5)$ & & \\
\hline & & & Mo-Mo & $0.5(1)$ & $3.35(1)$ & $0.001(1)$ & & \\
\hline
\end{tabular}


Table S2. Modelled EXAFS fitting parameters for $\mathrm{pH}_{0} 6.5$ experiments. Parameters include the amplitude reduction factor which was fixed at 0.82 , the Debye-Waller factor $\left(\sigma^{2}\right)$, interatomic distance $(\mathrm{R})$, coordination number $(\mathrm{CN})$, and energy shift $\left(\Delta \mathrm{E}_{0}\right)$. The fitting range used was $\mathrm{k}=$ 3.0 to $11.0 \AA^{-1}$ and $\mathrm{R}=1.0$ to $4.0 \AA$. Time ' 0 ' indicates the experiments were collected before $\mathrm{Fe}(\mathrm{II})$ addition.

\begin{tabular}{|c|c|c|c|c|c|c|c|c|}
\hline pH & ID & Time & Path & $\mathbf{C N}$ & $\mathbf{R}(\AA)$ & $\sigma^{2}(\AA)$ & $\Delta \mathbf{E}_{0}$ & R-factor \\
\hline \multirow[t]{2}{*}{6.5} & Ads-Mo-L & 0 & Mo-O & $4.1(2)$ & $1.741(4)$ & $0.0024(6)$ & 5.18 & 0.004 \\
\hline & & & Mo-Fe & $0.7(6)$ & $2.78(2)$ & $0.009(7)$ & & \\
\hline \multirow[t]{2}{*}{6.5} & Ads-Mo-L & 168 & Mo-O & $3.2(3)$ & $1.726(6)$ & $0.0016(9)$ & 2.55 & 0.010 \\
\hline & & & $\mathrm{Mo}-\mathrm{Fe}_{2}$ & $0.6(5)$ & $3.15(2)$ & $0.001(60)$ & & \\
\hline \multirow[t]{2}{*}{6.5} & Ads-Mo-H & 0 & Mo-O & $4.0(2)$ & $1.740(4)$ & $0.0020(6)$ & 5.95 & 0.004 \\
\hline & & & $\mathrm{Mo}-\mathrm{Fe}_{1}$ & $0.4(4)$ & $2.76(3)$ & $0.006(8)$ & & \\
\hline \multirow[t]{2}{*}{6.5} & Ads-Mo-H & 168 & Mo-O & $3.1(2)$ & $1.727(5)$ & $0.0012(8)$ & 2.93 & 0.008 \\
\hline & & & $\mathrm{Mo}-\mathrm{Fe}_{2}$ & $0.5(4)$ & $3.2(2)$ & $0.001(6)$ & & \\
\hline \multirow[t]{2}{*}{6.5} & Cop-Mo-L & 0 & ${\mathrm{Mo}-\mathrm{O}_{1}}_{1}$ & $4.1(2)$ & $1.733(5)$ & $0.003(6)$ & 3.17 & 0.007 \\
\hline & & & $\mathrm{Mo}-\mathrm{Fe}_{2}$ & $0.5(1)$ & $3.17(2)$ & $0.001(1)$ & & \\
\hline \multirow[t]{5}{*}{6.5} & Cop-Mo-L & 168 & ${\mathrm{Mo}-\mathrm{O}_{1}}_{1}$ & $3.6(3)$ & $1.727(4)$ & $0.0048(8)$ & -3.21 & 0.010 \\
\hline & & & $\mathrm{Mo}-\mathrm{O}_{2}$ & $0.6(2)$ & $1.97(1)$ & $0.002(4)$ & & \\
\hline & & & $\mathrm{Mo}-\mathrm{Fe}_{2}$ & $1.3(1.3)$ & $3.02(3)$ & $0.01(1)$ & & \\
\hline & & & Mo-Mo & $1.2(6)$ & $3.33(1)$ & $0.004(3)$ & & \\
\hline & & & $\mathrm{Mo}-\mathrm{Fe}_{3}$ & $4.0(3.1)$ & $3.83(2)$ & $0.012(7)$ & & \\
\hline \multirow[t]{2}{*}{6.5} & Cop-Mo-H & 0 & ${\mathrm{Mo}-\mathrm{O}_{1}}_{1}$ & $3.6(2)$ & $1.732(5)$ & $0.0025(7)$ & 3.39 & 0.006 \\
\hline & & & $\mathrm{Mo}-\mathrm{Fe}_{2}$ & $0.6(4)$ & $3.142(5)$ & $0.001(5)$ & & \\
\hline \multirow[t]{5}{*}{6.5} & Cop-Mo-H & 168 & Mo-O & $3.0(1)$ & $1.715(2)$ & $0.0022(4)$ & -2.26 & 0.005 \\
\hline & & & Mo- $\mathrm{O}_{2}$ & $0.4(2)$ & $2.26(2)$ & $0.002(4)$ & & \\
\hline & & & $\mathrm{Mo}-\mathrm{Fe}_{2}$ & $1.2(6)$ & $3.07(1)$ & $0.006(4)$ & & \\
\hline & & & Mo-Mo & $1.0(3)$ & $3.346(4)$ & $0.002(2)$ & & \\
\hline & & & $\mathrm{Mo}-\mathrm{Fe}_{3}$ & $1.3(1.3)$ & $3.85(2)$ & $0.007(8)$ & & \\
\hline
\end{tabular}

